\title{
Neuromodulation via the Cerebrospinal Fluid: Insights from Recent in Vitro Studies
}

\author{
Andreas Bjorefeldt ${ }^{1,2 *}$, Sebastian Illes ${ }^{1}$, Henrik Zetterberg ${ }^{3,4,5,6}$ and Eric Hanse ${ }^{1}$ \\ ${ }^{1}$ Department of Physiology, Institute of Neuroscience and Physiology, University of Gothenburg, Gothenburg, Sweden, \\ ${ }^{2}$ Department of Neuroscience, Brown University, Providence, Rl, United States, ${ }^{3}$ Department of Psychiatry and \\ Neurochemistry, Institute of Neuroscience and Physiology, University of Gothenburg, Gothenburg, Sweden, \\ ${ }^{4}$ Clinical Neurochemistry Laboratory, Sahlgrenska University Hospital, Gothenburg, Sweden, ${ }^{5}$ Department of Molecular \\ Neuroscience, UCL Institute of Neurology, University College London, London, United Kingdom, ${ }^{6}$ United Kingdom Dementia \\ Research Institute, University College London, London, United Kingdom
}

\section{OPEN ACCESS}

Edited by:

Srikanth Ramaswamy,

École Polytechnique Fédérale

de Lausanne, Switzerland

Reviewed by: Graham William Knott,

École Polytechnique Fédérale

de Lausanne, Switzerland

Dasiel Oscar Borroto-Escuela,

Karolinska Institute (KI), Sweden

*Correspondence:

Andreas Bjorefeldt

andreas.bjorefeldt@gu.se

Received: 01 October 2017

Accepted: 11 January 2018

Published: 05 February 2018

Citation:

Bjorefeldt A, Illes S, Zetterberg H and Hanse $E$ (2018) Neuromodulation via the Cerebrospinal Fluid: Insights

from Recent in Vitro Studies.

Front. Neural Circuits 12:5.

doi: 10.3389/fncir.2018.00005
The cerebrospinal fluid (CSF) occupies the brain's ventricles and subarachnoid space and, together with the interstitial fluid (ISF), forms a continuous fluidic network that bathes all cells of the central nervous system (CNS). As such, the CSF is well positioned to actively distribute neuromodulators to neural circuits in vivo via volume transmission. Recent in vitro experimental work in brain slices and neuronal cultures has shown that human CSF indeed contains neuromodulators that strongly influence neuronal activity. Here we briefly summarize these new findings and discuss their potential relevance to neural circuits in health and disease.

Keywords: cerebrospinal fluid, neuromodulation, neural circuit

\section{INTRODUCTION}

The cerebrospinal fluid (CSF) system is an evolutionarily preserved feature of animal brains (Brocklehurst, 1979) and provides central neurons with a regulated chemical environment well suited to promote their function and survival. CSF is a clear transparent extracellular fluid occupying the ventricles (I-IV) and subarachnoid space (Figure 1A), and forms a continuous fluidic network together with the interstitial fluid (ISF) of the parenchyma (Figure 1B). Its composition is characterized by low protein $(\sim 1 \%$ of blood) and high salt $(>150 \mathrm{mmol} / \mathrm{L})$ content, and is influenced by multiple sources including blood, the choroid plexus, ventricular ependymal cells, neurons and glia (Smith et al., 2004; Skipor and Thiery, 2008).

Communicating freely with the ISF (Brightman and Palay, 1963; Smith et al., 2004), the CSF system has the potential to serve as a vessel for neuromodulatory signals acting via volume transmission (Agnati et al., 1986, 2010). However, whether the CSF system in fact plays an active, rather than simply passive, role in distributing neuromodulators throughout the brain is still unknown. In this review, we summarize a set of new experimental findings in vitro showing that endogenous neuromodulators in human CSF potently influence the function of pyramidal cells and interneurons in the rat hippocampal brain slice, and in rat cortical neuronal cultures. The potential significance of this neuromodulation is discussed in health and disease, and an outlook on the future advancement of this research field is provided. 

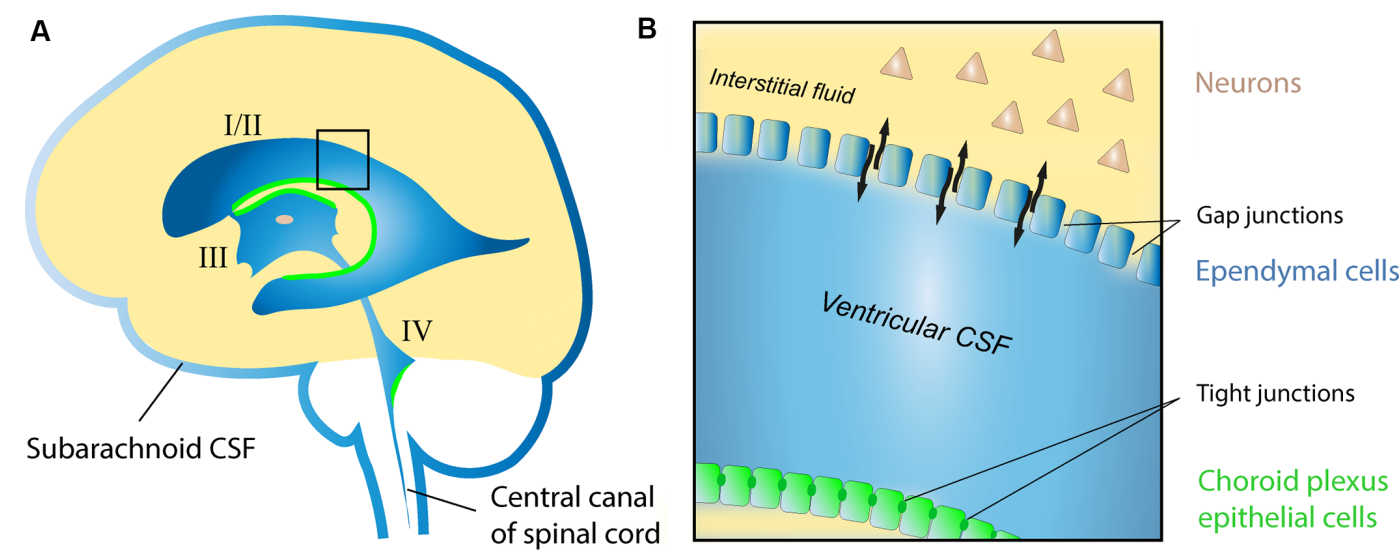

FIGURE 1 | Schematic drawing of the human CSF system. (A) The position of the lateral (I/II), third (III), and fourth (IV) ventricles with respective choroid plexa (green), and the subarachnoid CSF compartments surrounding the brain and spinal cord. (B) Enlargement of area highlighted in (A) showing the interface between ventricular CSF and the ISF of the parenchyma in the adult brain. Note the restricted molecular exchange across the choroid plexus epithelial lining, where cells are adjoined by tight junctions (blood-CSF barrier), as opposed to areas of the ventricular lining composed of ependymal cells connected by gap junctions (black arrows indicate free exchange of molecules between CSF and ISF at these sites).

\section{RECENT IN VITRO FINDINGS WITH HUMAN CEREBROSPINAL FLUID}

\section{Electrophysiological Recordings from Rat Hippocampal Brain Slices}

Since its introduction in the early 1970s, the hippocampal brain slice preparation has remained a major in vitro platform to study synaptic, cellular and network aspects of neuronal activity (Skrede and Westgaard, 1971). However, a limitation of this experimental model is that neurons in brain slices are perfused with artificial extracellular fluid (artificial CSF, aCSF) during recordings. Consisting simply of electrolytes, glucose and water, aCSF lacks the complex organic make-up of physiological CSF including proteins, peptides, lipids, amino acids, etc. Of particular relevance with respect to neuronal activity and function, physiological CSF is also known to contain a wide range of neuromodulators (Table 1) whose collective influence on cellular and synaptic properties has been poorly investigated.

In a recent study, Bjorefeldt et al. (2015) used the simplistic make-up of aCSF to establish the functional impact of endogenous neuromodulators in real brain extracellular fluid, i.e., human CSF (hCSF), on rat hippocampal neurons. The study measured electrolyte and glucose levels in pooled samples of hCSF obtained by lumbar puncture from both neurological patients and healthy volunteers. A matched aCSF was then designed based on obtained measurements and used as control for potential neuromodulatory effects of hCSF on hippocampal neurons. In whole-cell patch clamp recordings from CA1 pyramidal cells, hCSF caused a strong increase in neuronal excitability, boosting spontaneous action potential (AP) firing approximately fivefold (Figures 2A,B). Moreover, hCSF depolarized the resting membrane potential of CA1 pyramidal cells (Figures 2A,C) and lowered their firing threshold (Figures 2E,F) through apparent G-protein signaling-dependent mechanisms (Figures 2D,G), leading to a left shifted frequency-current (input-output) relationship (Figures 2H-J). In extracellular field recordings from CA3-CA1 synapses in stratum radiatum, hCSF caused a large increase in evoked excitatory synaptic transmission that was accompanied by an apparent increase in presynaptic release probability (Figure 2K). In attempt to isolate the active neuromodulatory fraction of hCSF based on molecular size, the authors dialyzed hCSF samples to remove all constituents larger than $8 \mathrm{kDa}$. Compared to untreated hCSF, evoked excitatory synaptic responses were strongly reduced in the dialyzed hCSF, indicating a large contribution from low molecular weight $(\leq 8 \mathrm{kDa})$ substances to these effects (Figure 2L). Overall the study revealed strong neuromodulatory influence of hCSF on hippocampal CA1 pyramidal cells, suggesting that such neuromodulation could be of relevance to pyramidal cell function in vivo.

Another recent study examined the effects of hCSF on GABAergic interneurons in CA1 stratum pyramidale of rat (Bjorefeldt et al., 2016). Cortical GABAergic interneurons are a diverse population consisting of many subtypes of cells with distinct anatomical and physiological properties (Klausberger and Somogyi, 2008; Moore et al., 2010; Tremblay et al., 2016). Bjorefeldt et al., 2016 examined two broad groups interneurons that were classified as having either fast-spiking (FS) or non-fast-spiking (NFS) phenotype based on their voltage response to a series of depolarizing and hyperpolarizing current injections (Bjorefeldt et al., 2016). The authors found that hCSF, again compared to a matched aCSF, increased the excitability of both groups of CA1 interneurons, boosting their spontaneous firing two-threefold over $10 \mathrm{~min}$ in whole-cell current clamp recordings (Figures 3A,B,D,I,J,L). However, in contrast to CA1 pyramidal cells, hCSF had no effect on the resting membrane 
TABLE 1 | Examples of neuromodulators present in CSF and their respective targets in the central nervous system.

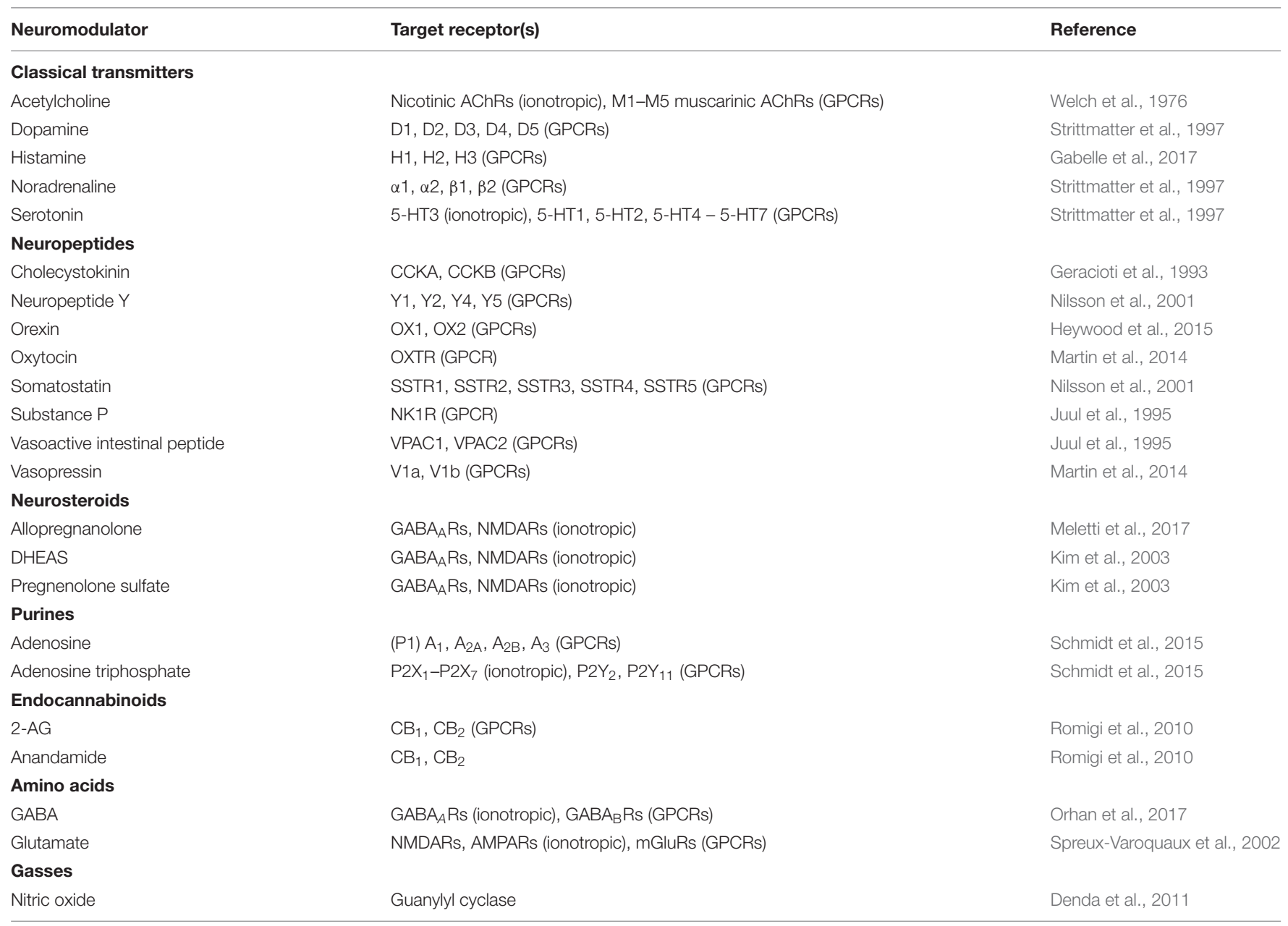

AChR, acetylcholine receptor; GPCR, G-protein coupled receptor; D1-5, dopamine receptors 1-5; H1-3, histamine receptors 1-3; $\alpha / \beta$ 1-2, alpha and beta noradrenergic receptors 1 and 2; 5-HT1-7, 5-hydroxytryptamine receptors 1-7; CCKA/B, cholecystokinin receptors $A$ and B; Y1, Y2, Y4, Y5, neuropeptide y receptors 1, 2, 4, and 5; OX1-2, orexin receptors 1 and 2; OXTR, oxytocin receptor; SSTR1-5, somatostatin receptors 1-5; NK1R; neurokinin 1 receptor; VPAC1-2, vasoactive intestinal peptide receptors 1 and 2; V1a/b, vasopressin receptors V1a and V1b; GABA $A_{A B} R$ s, types $A$ and B gamma-aminobutyric acid receptors; NMDARs, N-methyl-D-aspartic acid receptors; $A_{1}, A_{2 A} A_{B}, A_{3}$, adenosine receptors $A_{1}, A_{2 A}, A_{2 B}$ and $A_{3} ; P 2 X_{1-7} / P 2 Y_{2 / 11}$; adenosine triphosphate (ATP) receptors $P 2 X_{1-7}, P 2 Y_{2}$ and $P 2 Y_{11} ; C B_{1 / 2}$, cannabinoid receptors type 1 and 2; AMPARs, $\alpha$-amino-3-hydroxy-5-methyl-4-isoxazolepropionic acid receptors; mGluRs, metabotropic glutamate receptors.

potential of interneurons (Figures $\mathbf{3 B}, \mathbf{C}, \mathbf{J}, \mathbf{K}$ ), indicating cell specificity of the neuromodulatory effects. Similar to pyramidal cells, hCSF caused a left-shift in the frequencycurrent relationship of both groups of interneurons, increasing their responsiveness to excitatory input (Figures $3 \mathbf{E}-\mathbf{H}, \mathbf{M}-\mathbf{P}$ ). Moreover, both interneuron groups displayed increased firing in response to sinusoidal current injections at theta and gamma frequencies in hCSF. Together with strong excitation of pyramidal cells, these effects are likely to promote hippocampal oscillatory network activity (Bjorefeldt et al., unpublished findings).

Together these studies show that (i) hCSF contains physiologically active neuromodulators that potently increase the excitability of both pyramidal cells and interneurons in vitro, (ii) some of these neuromodulatory effects are cell-type specific and act through G-protein coupled receptors (GPCRs), (iii) pyramidal cells appear to be more strongly modulated than the two examined groups of GABAergic interneurons, (iv) active neuromodulators are largely $\leq 8 \mathrm{kDa}$ in size and (v) the presence of neuromodulators in physiological brain extracellular fluid such as hCSF can help explain differences in the amount of spontaneous neuronal activity observed in vivo vs. in typical in vitro brain slice recordings.

\section{Multielectrode Array Recordings from Rodent Primary and Stem Cell-Derived Neural Cultures}

Multielectrode arrays (MEAs) are extracellular recording devices allowing the detection of neuronal network activity generated by cultured neurons or within brain slice preparations (Figure 4). MEAs are composed of tens of electrodes embedded into a glass or plastic substrate. On this electrode array, neural cells can be cultured and brain slice preparations can be placed to study neuronal activity at the single cell and network level for minutes up to several months (Obien et al., 2014). 


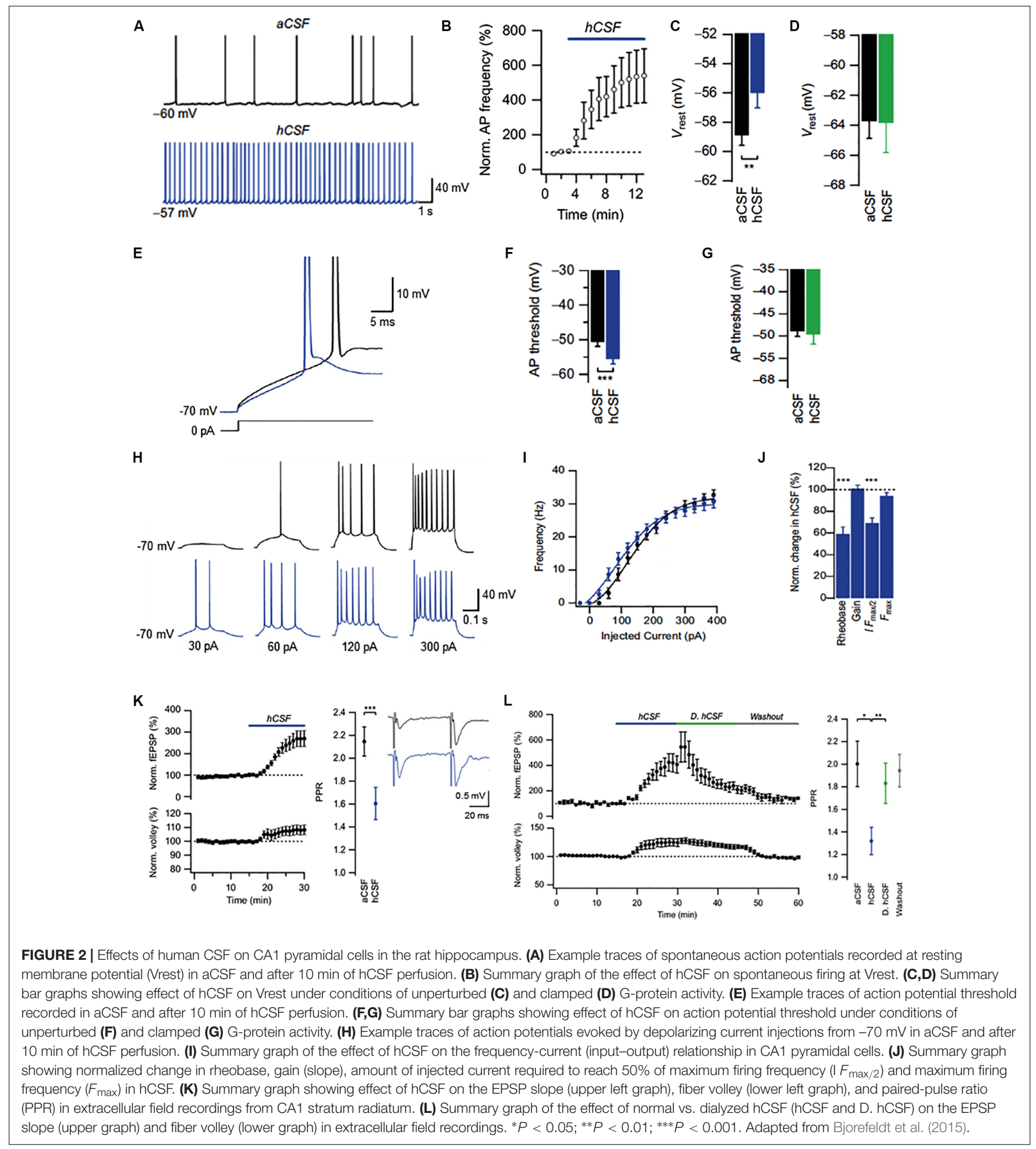

For this purpose, MEAs are connected to an amplifier and computer system, where an increasing amount of data analysis approaches is being used to analyze the electrophysiological neuronal activity. The extracted neuronal signals are comprised of spikes (filtered signals $>200 \mathrm{~Hz}$ ) and local field potentials (filtered signals $<200 \mathrm{~Hz}$ ), and thereby, neuronal function can be analyzed at different levels in a non-invasive approach. As the extracellular solution in the recording chamber is easily exchangeable, MEAs represent an ideal non-invasive measurement tool to understand the acute and chronic impact of CSF samples on single neurons as well as neuronal networks. 


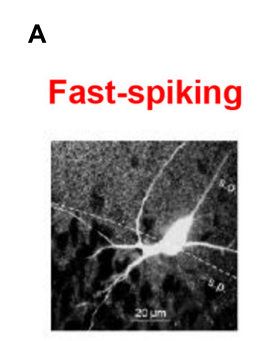

$\mathbf{E}$
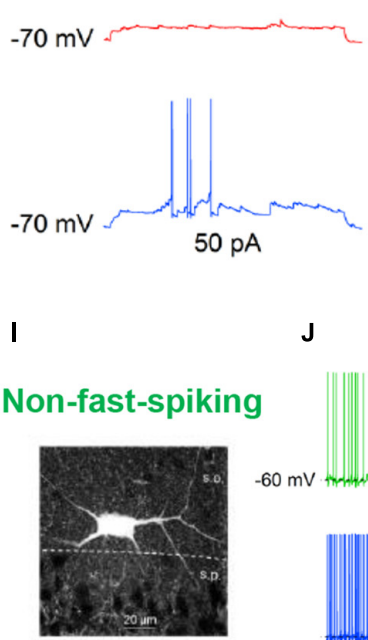

$\mathbf{J}$

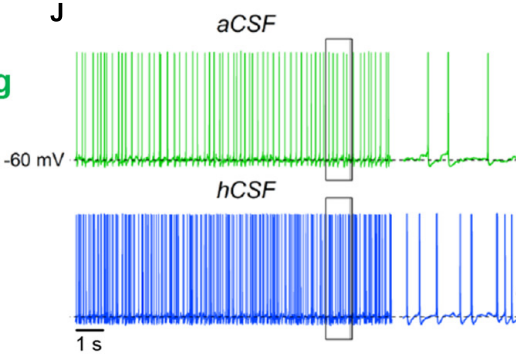

M

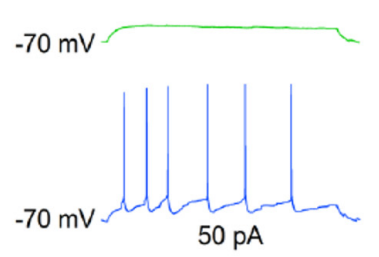

B

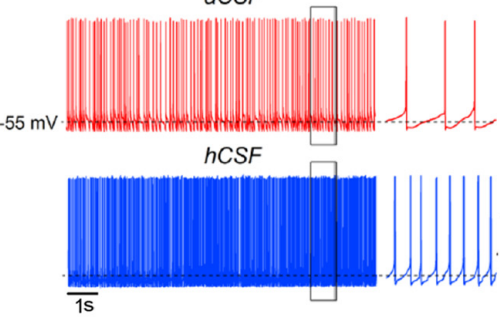

F

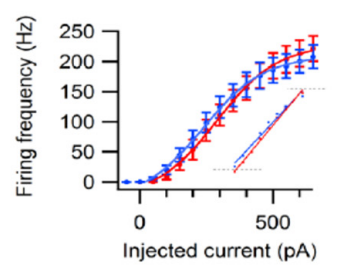

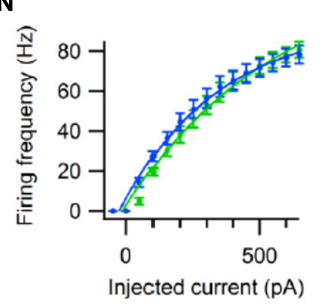

C

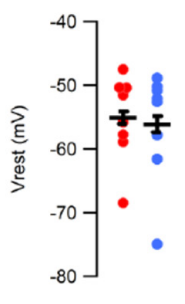

D

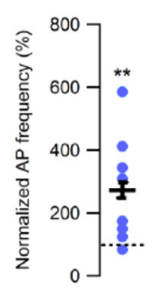

H

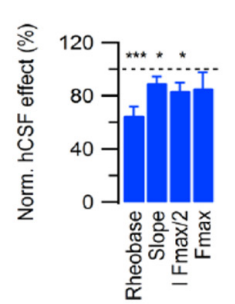

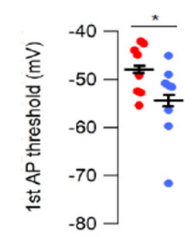

K

L
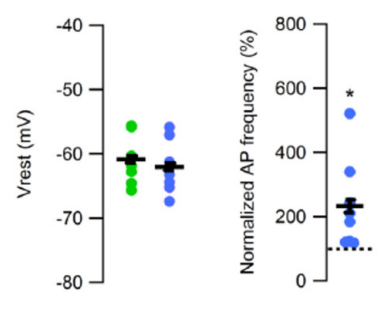

P

0

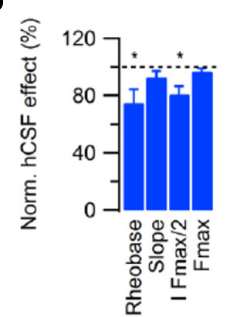

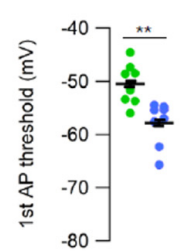

FIGURE 3 | Effects of human CSF on fast-spiking and non-fast-spiking rat hippocampal CA1 interneurons. (A) Example of cell morphology in the FS interneuron group. (B) Example traces of spontaneous action potentials recorded at Vrest in aCSF and after 10 min of hCSF perfusion in a FS interneuron. (C,D) Summary bar graphs showing effect of hCSF on Vrest (C) and spontaneous firing frequency (D) in the FS interneuron group. (E) Example traces of action potentials evoked by depolarizing current injection from $-70 \mathrm{mV}$ in aCSF and after $10 \mathrm{~min}$ of hCSF perfusion. (F) Summary graph of the effect of hCSF on the frequency-current (input-output) relationship in FS CA1 interneurons. (G) Summary graph showing normalized change in rheobase, gain (slope), amount of injected current required to reach $50 \%$ of maximum firing frequency $\left(I F_{\max / 2}\right)$ and maximum firing frequency $\left(F_{\max }\right)$ in hCSF. $(\mathbf{H})$ Summary graph of the effect of hCSF on the threshold of first elicited action potential in response to depolarizing current injection from $-70 \mathrm{mV}$ in FS interneurons. (I) Example of cell morphology in the NFS interneuron group. (J) Example traces of spontaneous action potentials recorded at Vrest in aCSF and after 10 min of hCSF perfusion in a NFS interneuron. (K,L) Summary bar graphs showing effect of hCSF on Vrest (K) and spontaneous firing frequency (L) in the NFS interneuron group. (M) Example traces of action potentials evoked by depolarizing current injection from $-70 \mathrm{mV}$ in aCSF and after $10 \mathrm{~min}$ of hCSF perfusion. (N) Summary graph of the effect of hCSF on the frequency-current (input-output) relationship in NFS CA1 interneurons. (O) Summary graph showing normalized change in rheobase, gain (slope), amount of injected current required to reach $50 \%$ of maximum firing frequency $\left(I F_{\max / 2}\right)$ and maximum firing frequency $\left(F_{\max }\right)$ in hCSF. (P) Summary graph of the effect of hCSF on the threshold of first elicited action potential in response to depolarizing current injection from $-70 \mathrm{mV}$ in NFS interneurons. ${ }^{*} P<0.05$; ${ }^{* *} P<0.01$; ${ }^{* * *} P<0.001$. Adapted from Bjorefeldt et al. (2016).

Otto et al. (2009) described the neuronal network function of rodent primary tissue-derived, and stem cell-derived, neurons exposed to aCSF or to hCSF samples obtained from healthy individuals and traumatic brain injury (TBI) patients by applying MEAs. The mouse embryonic stem cell (mESC)-derived neuronal population used comprises $\sim 70 \%$ GABAergic neurons (Illes et al., 2009) and $\sim 70 \%$ of neurons are intrinsically active (Illes et al., 2014). Due to this cellular composition, the mESC-derived neuronal cultures are predominantly asynchronously or partially synchronously 
multielectrode array (MEA) and extracellular recordinas from cortical neuronal networks

A

B

C
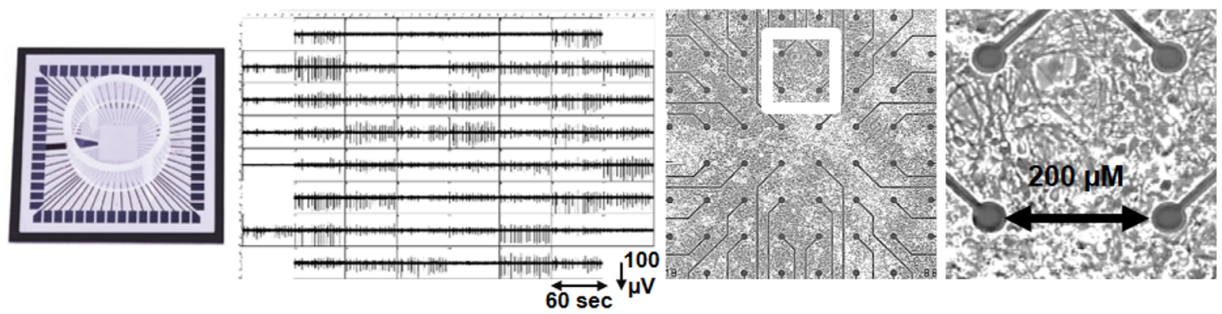

D

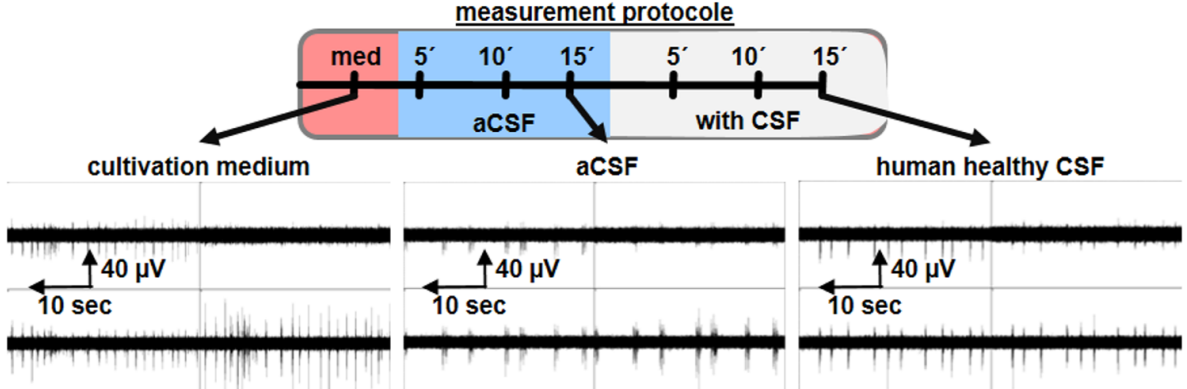

FIGURE 4 | Microelectrode array recordings of neuronal culture to study the impact of human CSF on neuronal network activity. Representative image of a (A) multielectrode array (MEA), (B) synchronous neuronal network activity detected by 59 electrodes, (C) cortical neural cells cultures on MEAs, and cortical neuronal morphology. (D) Schematic drawing of a recording procedure used to study the functional impact of human CSF samples on neuronal cultures. Examples show the neuronal network activity of cortical neurons exposed to cultivation media, aCSF and human CSF samples from a healthy individual.

active in aCSF or cultivation media. However, substitution of aCSF with healthy hCSF produces highly synchronous neuronal network activity. As described by Otto et al. (2009), several network activity parameters are increased after healthy hCSF application, such as the number of spikes, population bursts and parameters to describe synchrony such as Cohen's kappa value. Since hippocampal and cortical rodent neuronal cultures are predominantly of glutamatergic identity, the recorded network activity in the presence of culture media or aCSF is highly synchronous (Wagenaar et al., 2006; Illes et al., 2014; Perez-Alcazar et al., 2016) (Figure 4D). Nevertheless, hippocampal or cortical neuronal cultures exposed to healthy hCSF show a strong increase in the number of population bursts (Otto et al., 2009; Gortz et al., 2013) (Figure 4D). Thus, all together these studies show that hCSF enhances neuronal activity and induces synchronicity in the network (Otto et al., 2009; Gortz et al., 2013; Jantzen et al., 2013), which is consistent with the results obtained in hippocampal brain slices.

\section{Study Limitations}

There are a number of outstanding questions and limitations associated with above described studies. Perhaps most notable is that the specific identity of active neuromodulators in hCSF, as well as their respective receptor signaling pathways, remains to be identified. A further limitation is that effects of hCSF were examined in neurons from rat. Thus, to what extent are findings with hCSF in these studies of relevance to the human brain? To control for potential species differences in neuromodulatory effects we attempted to sample CSF from the cisterna magna of rats. Unfortunately, given the small extractable volume from each rat $(\sim 150 \mu \mathrm{L})$ we were unable to obtain a CSF pool large enough to allow proper electrophysiological experimentation. Since neuromodulatory systems architecture is known to be well conserved across species (O'Connell, 2013; Swallow et al., 2016; Lovett-Barron et al., 2017), we would expect hCSF to produce similar effects on human hippocampal and cortical neurons. In line with this prediction, a recent study using human neocortical brain slices prepared from resected tissue of epilepsy patients reported that hCSF, as compared to artificial culturing media, enhanced neuronal activity (Schwarz et al., 2017).

Another issue relates to the artificial manner in which neurons were exposed to neuromodulators in these studies. hCSF was introduced directly to brain slices and cultured neurons via an artificial perfusion system, allowing neuromodulators to bypass any natural diffusion barrier. It therefore remains unclear whether certain neuromodulators in hCSF would ever reach the corresponding areas in the intact brain. Moreover, it is possible that the neuromodulatory composition of hCSF could differ between brain and spinal compartments. All hCSF used in above described studies was sampled from the lumbar subarachnoid space, raising the question of whether hCSF sampled, e.g., from one of the ventricles could have produced somewhat different effects.

Finally, a number of studies suggest that the CSF system during development is more compartmentalized than in the 
adult and young adult brain due to the presence of strap junctions between ependymal cells that largely limit the exchange of molecules between the CSF and ISF (Saunders et al., 2000; Whish et al., 2015). Thus, it should be noted that the findings with adult hCSF in above described studies may not have significant implications for neuromodulatory CSF signaling during development.

\section{PROPERTIES AND POTENTIAL SIGNIFICANCE OF CSF NEUROMODULATION}

The essential finding that hCSF contains physiologically active neuromodulators at ambient levels can be interpreted in light of two separate, although not mutually exclusive, scenarios. One possibility is that the neuromodulators, whose specific identities and relative functional importance are yet to be fully established, were selectively secreted into the CSF system in order to exert effects at downstream target areas. In this case, one could consider the CSF as an 'active' vessel or distributor of neuromodulatory signals within the brain, as has been previously suggested (Skipor and Thiery, 2008; Veening and Barendregt, 2010; Fuxe et al., 2012). An alternative scenario, considering the free bidirectional exchange of molecules between the CSF and ISF, is that the CSF acts as a sink for neuromodulatory substances originating from the neuronal parenchyma, diffusing away from their site of release along their concentration gradients. The CSF could then be viewed as reflecting an overall neuromodulatory environment with broad spatiotemporal profile, perhaps merely transporting neuromodulators that are to be cleared from the brain parenchyma.

Whether neuromodulators are in fact actively secreted into the CSF in vivo or not, their remarkably potent effects on cortical neurons in recent studies have interesting implications for neuromodulation and neuronal communication in the brain. These findings show that (i) there exists neuromodulatory substances in brain extracellular fluid that retain physiological function over prolonged time periods (up to several hours in our studies) and (ii) there are neuromodulatory GPCRs (not excluding contributions from other receptor types) in cortex with sufficient affinity for these neuromodulators to mediate observed effects on neuronal activity. Thus, these results provide strong support for the concept of long distance (non-synaptic) volume transmission (Agnati et al., 1986, 2010) as a means for neuronal communication in the brain. In line with our findings, neuromodulators acting via CSF volume transmission in vivo would likely operate on a slow (minutes to hours) timescale with broad spatial distribution, targeting neuromodulatory GPCR families (Fuxe et al., 2012, 2013). In addition to canonical intracellular metabotropic signaling, GPCR activation may influence ionotropic receptor families via heterodimerization (Borroto-Escuela et al., 2010, 2017).

Given the wide range of neuromodulators found in CSF (see Table 1), their collective influence on neural circuits should be considered. In such a paradigm, the functional impact of a given neuromodulator would depend on its local concentration, target receptor affinity, expression and distribution, as well as sensitivity to enzymatic degradation and/or uptake by transporter proteins. Moreover, parameters affecting diffusion through extracellular space, such as local tissue tortuosity and chemical properties of the neuromodulator (e.g., molecular weight and charge distribution), are likely to be important (Sykova, 2004). An interesting aspect of multiple neuromodulators acting via volume transmission to exert simultaneous influence on neural circuits is the potential for synergistic neuromodulatory effects, e.g., through converging intracellular signaling pathways.

Our recent findings have indicated that a major functional effect of CSF-distributed neuromodulators on cortical neurons is to boost intrinsic excitability and increase neuronal responsiveness to excitatory input. It is possible that these effects, causing a left-shift in neuronal input-output function, are indicative of how neuromodulation directs neurons and circuits into an efficient 'online' information processing state during wakefulness. The neuromodulatory effects of hCSF could facilitate and/or support multiple aspects of neural circuit function in vivo. For example, allowing fewer numbers of simultaneously active synapses to drive AP output would support a sparse and energy efficient information coding regime, which is thought to be utilized by the brain (Olshausen and Field, 2004; Wolfe et al., 2010; Barth and Poulet, 2012; Palm, 2013). With respect to learning and memory, the increased excitability of cortical pyramidal cells is likely to enhance NMDA receptor-mediated coincidence detection and synaptic plasticity in neural circuits through membrane potential depolarization and increased numbers of backpropagating APs (Tsubokawa and Ross, 1997; Lisman and Spruston, 2005). This would promote learning-associated structural and functional modifications to neural circuits during active wake, which is when synapses are thought to undergo strengthening to support acquisition and storage of new information (Tononi and Cirelli, 2014). In cortical networks, periods of synchronized fast GABAergic inhibition onto principal cells give rise to rhythmic membrane potential fluctuations in neuronal populations (network oscillations) that are hypothesized to facilitate information processing and cognition (Singer, 1999; Buzsaki, 2006; Lisman and Jensen, 2013). By boosting the excitability of both GABAergic interneurons and principal cells, it is likely that CSF neuromodulation would promote such rhythm generation in cortical networks and circuits.

In summary, in terms of its functional effects on neurons, this type of neuromodulation seems to support multiple circuit mechanisms hypothesized to underlie cognitive abilities. As an ambient form of neuromodulation it may serve to promote and/or facilitate signature neuronal activity patterns important in information coding and processing by neural circuits. Neuromodulatory effects of other spatiotemporal characteristics would be able to operate on top of such an ambient component, further enriching the means by which neuromodulation can regulate neural circuit function. 


\section{ORIGIN AND IDENTITY OF CSF NEUROMODULATORS}

The chemical composition of CSF is shaped by contributions from multiple sources including blood, the choroid plexus, ependymal cells, neurons and glia (Smith et al., 2004; Skipor and Thiery, 2008). With respect to neuromodulators, release from CNS resident cells is likely to be a major source but the relative contribution of different CNS compartments is largely unknown. Part of the neuromodulatory composition of CSF could result from the tonic activity of subcortical monoaminergic and cholinergic nuclei during wakefulness, resulting in widespread release of noradrenaline, histamine, dopamine and serotonin and acetylcholine throughout the brain (Saper et al., 2001). The finding that release often occurs from varicosities lacking a postsynaptic element (Descarries and Mechawar, 2000; Vizi et al., 2010), and that there is mismatch in transmitter release site vs. receptor location (Jansson et al., 2002; Fuxe et al., 2005), suggests that monoamines and acetylcholine operate as volume transmitters in the CNS. Depending on local regulation of reuptake mechanisms and acetylcholine esterase levels, these classical transmitters could be active over long distances. Another potential source is the CSF-contacting neurons found in periventricular brain regions of a wide range of species. Some of these neurons feature varicose neuromodulator-containing processes that terminate in ventricular CSF (Parent, 1981; Vigh et al., 2004; Veening and Barendregt, 2010), suggesting that neuromodulators may be actively released into the CSF to exert downstream effects on target neurons. Dendritic neuropeptide release from large densecore vesicles, such as seen in hypothalamic neuronal populations (Ludwig and Leng, 2006), may additionally contribute to the neuromodulatory composition of CSF. In addition to CNS derived release, certain blood-borne neuromodulators such as leptin have been shown to enter the CSF by way of specific transporters expressed at the choroid plexus bloodCSF barrier (Zlokovic et al., 2000; Skipor and Thiery, 2008). Furthermore, choroid plexus epithelial cells are known to secrete certain neuropeptides and neurotrophic factors into the CSF (Chodobski and Szmydynger-Chodobska, 2001; Smith et al., 2004).

Our work so far has focused largely on characterizing the functional impact of CSF neuromodulators on cortical neurons. While we believe this to be an important effort, the specific identities of active neuromodulators in hCSF should also be thoroughly addressed. There is at present evidence suggesting critical contribution from a distinct receptor subfamily in promoting hippocampal network oscillations in vitro (Bjorefeldt et al., unpublished findings). Depending on the experimental readout of neuronal activity (synaptic, cellular, network), and the type of neuronal population or brain area examined, the relative importance of specific neuromodulators in CSF and their various receptor signaling pathways may vary.

It can be concluded that any neuromodulator remaining functionally active at the level observed in present studies is remarkably resistant to both in vivo degradation and reuptake, and experimental handling such as thawing and freezing.
Neuropeptides may display long half-lives in brain extracellular fluid and are active at nanomolar concentrations due to their high affinity GPCRs (Jones and Robinson, 1982; Ludwig and Leng, 2006; van den Pol, 2012). The actions of peripherally secreted peptides such as leptin and ghrelin on central neurons further suggest plausibility of neuropeptide involvement in the neuromodulatory effects of hCSF. Interestingly, some neuropeptides have also been shown to enhance effects of nonpeptide neuromodulators such as acetylcholine (Mancillas et al., 1986; Kouznetsova and Nistri, 2000). Whether such results exemplify synergistic or additive neuromodulatory effects, this provides a mechanism by which multiple neuromodulators present at low ambient concentrations could significantly influence the operation of neural circuits in vivo.

\section{CSF NEUROMODULATORS IN DISEASE}

The most studied neuromodulators in CSF in relation to disease are the classical transmitters dopamine, serotonin, acetylcholine, histamine, and noradrenaline. A prototype neurodegenerative disease in which neuromodulation is impaired is Parkinson's disease; neurodegeneration-mediated depletion of dopamine in the striatum (especially the putamen) is the defining feature of the disorder, which has translated into a clinically established therapy based on levodopa/carbidopa treatment (Carlsson, 2002). Given the dopaminergic lesion, measurements of CSF concentrations of dopamine or its metabolites should provide a relatively straightforward diagnostic test but so far this expectation has not been realized. Nevertheless, some reports have noted decreased homovanillic acid (HVA, the end-product of dopamine metabolism) concentrations in CSF from patients with Parkinson's disease (Zubenko et al., 1986; Chia et al., 1993; Loeffler et al., 1995), or decreased CSF concentrations of dihydroxyphenylacetic acid (DOPAC; the main neuronal metabolite of dopamine) (Zubenko et al., 1986; Chia et al., 1993; Gonzalez-Quevedo et al., 1993; Eldrup et al., 1995). These results were not confirmed in the deprenyl and tocopherol antioxidative treatment of parkinsonism (DATATOP) trial that reported negative results for both CSF HVA and DOPAC in Parkinson's disease (LeWitt et al., 1992).

There are many potential reasons for why dopamine metabolite concentrations in CSF are not robustly associated with Parkinson's disease. CSF HVA is only distantly related to neuronal dopamine stores and reflects several intervening processes. Since dopaminergic neurons do not contain catechol-Omethyltransferase, CSF HVA depends on uptake and intracellular $O$-methylation in non-dopaminergic cells. Thus, in Parkinson's disease, the striatal content of HVA is not as severely decreased as that of dopamine (Lloyd et al., 1975).

Cerebrospinal fluid dopamine concentration may also not be a direct marker of central dopamine deficiency; increased dopamine release from remaining nerve terminals may compensate the loss of dopaminergic neurons in Parkinson's disease, thereby augmenting dopamine delivery from those terminals to the extracellular fluid (Sossi et al., 2002). CSF 
dopamine concentration may thus underestimate the extent of loss of neuronal dopamine stores.

Cerebrospinal fluid DOPAC may be a superior marker of central dopamine stores, compared to HVA or dopamine itself. DOPAC is formed from deamination of cytosolic dopamine catalyzed by monoamine oxidase-A. Dopamine leaks continuously from vesicular stores into the cytosol. Therefore, the rate of DOPAC formation should be related to the amount of stored dopamine. Post-mortem putamen from patients with end-stage Parkinson's disease shows similarly decreased tissue concentrations of dopamine and DOPAC (Goldstein et al., 2011), and CSF DOPAC is related directly to brain tissue content of this metabolite (Palfreyman et al., 1982).

Although several reports have noted low CSF DOPAC in Parkinson's disease, these were relatively small studies (Goldstein, 2013). Further, they did not compare CSF DOPAC levels in other neurodegenerative diseases and it is presently unclear whether CSF DOPAC reduction is specific to Parkinson's disease or not. More research on these topics is needed.

Other diseases in which neuromodulators play a major role for the clinical presentation are narcolepsia (orexin), Alzheimer's disease (acetylcholine), neuroinflammatory conditions, including multiple sclerosis (cytokines and interleukins), schizophrenia (dopamine and its metabolites) and affective disorders (serotonin and its metabolites). CSF orexin concentration is used as an adjunct in the criteria of the disease [CSF orexin concentration is low in the disease due to the loss of orexin-producing neurons (Bourgin et al., 2008)]. For Alzheimer's disease and affective disorders, changes in the neuromodulator concentrations are not specific enough to be clinically meaningful.

\section{TOWARD A BETTER UNDERSTANDING OF THE ROLE OF THE CSF SYSTEM IN NEUROMODULATION}

Neuromodulation encompasses a vast variety of biochemical processes, occurring at various spatiotemporal scales, which tune the excitability and function of central neurons. In this review, we have considered how long distance neuromodulation via the CSF might influence the function of neurons and neural circuits in health and disease, in light of new experimental findings. Further efforts are needed in order to establish whether the CSF acts as an 'active' neuromodulatory channel in vivo and, if so, how this

\section{REFERENCES}

Agnati, L. F., Fuxe, K., Zoli, M., Ozini, I., Toffano, G., and Ferraguti, F. (1986). A correlation analysis of the regional distribution of central enkephalin and beta-endorphin immunoreactive terminals and of opiate receptors in adult and old male rats. Evidence for the existence of two main types of communication in the central nervous system: the volume transmission and the wiring transmission. Acta Physiol. Scand. 128, 201-207. doi: 10.1111/j.1748-1716.1986. tb07967.x

Agnati, L. F., Guidolin, D., Guescini, M., Genedani, S., and Fuxe, K. (2010). Understanding wiring and volume transmission. Brain Res. Rev. 64, 137-159. doi: 10.1016/j.brainresrev.2010.03.003 neuromodulation impacts neural circuit function, e.g., during different brain states such as sleep and wake. To experimentally confirm such active role of the CSF system would require in vivo demonstration of (i) active release of neuromodulator into a CSF compartment, (ii) neuromodulator utilization of the CSF/ISF system to distribute within the brain and (iii) a functional impact of the neuromodulator(s) on a distant neuronal population. Moreover, it would require the capacity to distinguish between functional effects caused by CSF-derived and locally released neuromodulator.

A conceivable path toward elucidating the role of the CSF system in neuromodulation involves further research into the brain's CSF-contacting neurons. Identification of cell type-specific markers enabling selective manipulation of CSF-contacting neurons that harbor neuromodulatory release machinery would greatly aid in this effort. The development of better techniques for detecting neuromodulator release and diffusion in vivo combined with use of, e.g., fluorescent GPCR activation reporters and genetically encoded calcium/voltage indicators should provide new and better means to address the functional relevance of neuromodulation via the CSF system in health and disease. At present, further in vitro studies examining the neuromodulatory influence of healthy and pathological CSF on central neurons and circuits are warranted.

\section{AUTHOR CONTRIBUTIONS}

All authors took part in designing, writing and revising the manuscript. $\mathrm{AB}$ and SI provided the data and prepared the figures and tables.

\section{ACKNOWLEDGMENTS}

This work was supported by research grants issued by the Swedish Research Council (2016-06760, AB; 2013-2546, HZ; 2016-00986, EH), Swedish State Support for Clinical Research (ALFGBG-441051, HZ; ALFGBG-427611, EH), Alzheimerfonden (AF-556051/AF-744871, SI; AF-640391, EH), the Åke Wisberg Foundation (M17-0265, SI), the Swedish Brain Foundation (F02017-0035, EH), the European Research Council (681712, HZ) and the Frederik and Ingrid Thuring Foundation (2016-0225, SI).

Barth, A. L., and Poulet, J. F. (2012). Experimental evidence for sparse firing in the neocortex. Trends Neurosci. 35, 345-355. doi: 10.1016/j.tins.2012. 03.008

Bjorefeldt, A., Andreasson, U., Daborg, J., Riebe, I., Wasling, P., Zetterberg, H., et al. (2015). Human cerebrospinal fluid increases the excitability of pyramidal neurons in the in vitro brain slice. J. Physiol. 593, 231-243. doi: 10.1113/jphysiol. 2014.284711

Bjorefeldt, A., Wasling, P., Zetterberg, H., and Hanse, E. (2016). Neuromodulation of fast-spiking and non-fast-spiking hippocampal CA1 interneurons by human cerebrospinal fluid. J. Physiol. 594, 937-952. doi: 10.1113/JP271553

Borroto-Escuela, D. O., Carlsson, J., Ambrogini, P., Narvaez, M., Wydra, K., Tarakanov, A. O., et al. (2017). Understanding the role of GPCR heteroreceptor 
complexes in modulating the brain networks in health and disease. Front. Cell Neurosci. 11:37. doi: 10.3389/fncel.2017.00037

Borroto-Escuela, D. O., Narvaez, M., Marcellino, D., Parrado, C., Narvaez, J. A., Tarakanov, A. O., et al. (2010). Galanin receptor-1 modulates 5hydroxtryptamine-1A signaling via heterodimerization. Biochem. Biophys. Res. Commun. 393, 767-772. doi: 10.1016/j.bbrc.2010.02.078

Bourgin, P., Zeitzer, J. M., and Mignot, E. (2008). CSF hypocretin-1 assessment in sleep and neurological disorders. Lancet Neurol. 7, 649-662. doi: 10.1016/ S1474-4422(08)70140-6

Brightman, M. W., and Palay, S. L. (1963). The Fine Structure of Ependyma in the Brain of the Rat. J. Cell Biol. 19, 415-439. doi: 10.1083/jcb.19. 2.415

Brocklehurst, G. (1979). The significance of the evolution of the cerebrospinal fluid system. Ann. R. Coll. Surg. Engl. 61, 349-356.

Buzsaki, G. (2006). Rhythms of the Brain. Oxford: Oxford University Press. doi: 10.1093/acprof:oso/9780195301069.001.0001

Carlsson, A. (2002). Treatment of Parkinson's with L-DOPA. The early discovery phase, and a comment on current problems. J. Neural. Transm. 109, 777-787. doi: $10.1007 /$ s007020200064

Chia, L. G., Cheng, F. C., and Kuo, J. S. (1993). Monoamines and their metabolites in plasma and lumbar cerebrospinal fluid of Chinese patients with Parkinson's disease. J. Neurol. Sci. 116, 125-134. doi: 10.1016/0022-510X(93) 90316-Q

Chodobski, A., and Szmydynger-Chodobska, J. (2001). Choroid plexus: target for polypeptides and site of their synthesis. Microsc. Res. Tech. 52, 65-82. doi: 10.1002/1097-0029(20010101)52:1<65::AID-JEMT9>3.0.CO;2-4

Denda, H., Kimura, S., Yamazaki, A., Hosaka, N., Takano, Y., Imura, K., et al. (2011). Clinical significance of cerebrospinal fluid nitric oxide concentrations in degenerative cervical and lumbar diseases. Eur. Spine J. 20, 604-611. doi: 10.1007/s00586-010-1663-8

Descarries, L., and Mechawar, N. (2000). Ultrastructural evidence for diffuse transmission by monoamine and acetylcholine neurons of the central nervous system. Prog. Brain Res. 125, 27-47. doi: 10.1016/S0079-6123(00)25 $005-\mathrm{X}$

Eldrup, E., Mogensen, P., Jacobsen, J., Pakkenberg, H., and Christensen, N. J. (1995). CSF and plasma concentrations of free norepinephrine, dopamine, 3,4dihydroxyphenylacetic acid (DOPAC), 3,4-dihydroxyphenylalanine (DOPA), and epinephrine in Parkinson's disease. Acta Neurol. Scand. 92, 116-121. doi: 10.1111/j.1600-0404.1995.tb01023.x

Fuxe, K., Borroto-Escuela, D. O., Romero-Fernandez, W., Diaz-Cabiale, Z., Rivera, A., Ferraro, L., et al. (2012). Extrasynaptic neurotransmission in the modulation of brain function. Focus on the striatal neuronal-glial networks. Front. Physiol. 3:136. doi: 10.3389/fphys.2012.00136

Fuxe, K., Borroto-Escuela, D. O., Romero-Fernandez, W., Zhang, W. B., and Agnati, L. F. (2013). Volume transmission and its different forms in the central nervous system. Chin J. Integr. Med. 19, 323-329. doi: 10.1007/s11655-0131455- 1

Fuxe, K., Rivera, A., Jacobsen, K. X., Hoistad, M., Leo, G., Horvath, T. L., et al. (2005). Dynamics of volume transmission in the brain. Focus on catecholamine and opioid peptide communication and the role of uncoupling protein 2. J. Neural Transm. 112, 65-76. doi: 10.1007/s00702-0040158-3

Gabelle, A., Jaussent, I., Hirtz, C., Vialaret, J., Navucet, S., Grasselli, C., et al. (2017). Cerebrospinal fluid levels of orexin-A and histamine, and sleep profile within the Alzheimer process. Neurobiol. Aging 53, 59-66. doi: 10.1016/j. neurobiolaging.2017.01.011

Geracioti, T. D. Jr., Nicholson, W. E., Orth, D. N., Ekhator, N. N., and Loosen, P. T. (1993). Cholecystokinin in human cerebrospinal fluid: concentrations, dynamics, molecular forms and relationship to fasting and feeding in health, depression and alcoholism. Brain Res. 629, 260-268. doi: 10.1016/00068993(93)91329-Q

Goldstein, D. S. (2013). Biomarkers, mechanisms, and potential prevention of catecholamine neuron loss in Parkinson disease. Adv. Pharmacol. 68, 235-272. doi: 10.1016/B978-0-12-411512-5.00012-9

Goldstein, D. S., Holmes, C., Kopin, I. J., and Sharabi, Y. (2011). Intraneuronal vesicular uptake of catecholamines is decreased in patients with Lewy body diseases. J. Clin. Invest. 121, 3320-3330. doi: 10.1172/JCI4 5803
Gonzalez-Quevedo, A., Garcia, J. C., Fernandez, R., and Fernandez Cartaya, L. (1993). Monoamine metabolites in normal human cerebrospinal fluid and in degenerative diseases of the central nervous system. Bol. Estud. Med. Biol. 41, 13-19.

Gortz, P., Siebler, M., Ihl, R., Henning, U., Luckhaus, C., Supprian, T., et al. (2013). Multielectrode array analysis of cerebrospinal fluid in Alzheimer's disease versus mild cognitive impairment: a potential diagnostic and treatment biomarker. Biochem. Biophys. Res. Commun. 434, 293-297. doi: 10.1016/j.bbrc. 2013.02.121

Heywood, W. E., Galimberti, D., Bliss, E., Sirka, E., Paterson, R. W., Magdalinou, N. K., et al. (2015). Identification of novel CSF biomarkers for neurodegeneration and their validation by a high-throughput multiplexed targeted proteomic assay. Mol. Neurodegener. 10:64. doi: 10.1186/s13024-0150059-y

Illes, S., Jakab, M., Beyer, F., Gelfert, R., Couillard-Despres, S., Schnitzler, A., et al. (2014). Intrinsically active and pacemaker neurons in pluripotent stem cellderived neuronal populations. Stem Cell Rep. 2, 323-336. doi: 10.1016/j.stemcr. 2014.01.006

Illes, S., Theiss, S., Hartung, H. P., Siebler, M., and Dihne, M. (2009). Nichedependent development of functional neuronal networks from embryonic stem cell-derived neural populations. BMC Neurosci. 10:93. doi: 10.1186/1471-2202$10-93$

Jansson, A., Descarries, L., Cornea-Herbert, V., Riad, M., Verge, D., Bancila, M., et al. (2002). "Transmitter-receptor mismatches in central dopamine, serotonin, and neuropeptide systems. Further evidence for volume transmission," in The Neuronal Environment: Brain Homeostasis in Health and Disease, ed. W. Walz (New York, NY: Humana Press), 83-101.

Jantzen, S. U., Ferrea, S., Wach, C., Quasthoff, K., Illes, S., Scherfeld, D., et al. (2013). In vitro neuronal network activity in NMDA receptor encephalitis. BMC Neurosci. 14:17. doi: 10.1186/1471-2202-14-17

Jones, P. M., and Robinson, I. C. (1982). Differential clearance of neurophysin and neurohypophysial peptides from the cerebrospinal fluid in conscious guinea pigs. Neuroendocrinology 34, 297-302. doi: 10.1159/000123316

Juul, R., Hara, H., Gisvold, S. E., Brubakk, A. O., Fredriksen, T. A., Waldemar, G., et al. (1995). Alterations in perivascular dilatory neuropeptides (CGRP, SP, VIP) in the external jugular vein and in the cerebrospinal fluid following subarachnoid haemorrhage in man. Acta Neurochir. 132, 32-41. doi: 10.1007/ BF01404845

Kim, S. B., Hill, M., Kwak, Y. T., Hampl, R., Jo, D. H., and Morfin, R. (2003). Neurosteroids: Cerebrospinal fluid levels for Alzheimer's disease and vascular dementia diagnostics. J. Clin. Endocrinol. Metab. 88, 5199-5206. doi: 10.1210/ jc.2003-030646

Klausberger, T., and Somogyi, P. (2008). Neuronal diversity and temporal dynamics: the unity of hippocampal circuit operations. Science 321, 53-57. doi: 10.1126/science.1149381

Kouznetsova, M., and Nistri, A. (2000). Facilitation of cholinergic transmission by substance P methyl ester in the mouse hippocampal slice preparation. Eur. J. Neurosci. 12, 585-594. doi: 10.1046/j.1460-9568.2000.00942.x

LeWitt, P. A., Galloway, M. P., Matson, W., Milbury, P., Mcdermott, M., Srivastava, D. K., et al. (1992). Markers of dopamine metabolism in Parkinson's disease. The Parkinson Study Group. Neurology 42, 2111-2117. doi: 10.1212/WNL.42. 11.2111

Lisman, J., and Spruston, N. (2005). Postsynaptic depolarization requirements for LTP and LTD: a critique of spike timing-dependent plasticity. Nat. Neurosci. 8, 839-841. doi: 10.1038/nn0705-839

Lisman, J. E., and Jensen, O. (2013). The theta-gamma neural code. Neuron 77, 1002-1016. doi: 10.1016/j.neuron.2013.03.007

Lloyd, K. G., Davidson, L., and Hornykiewicz, O. (1975). The neurochemistry of Parkinson's disease: effect of L-dopa therapy. J. Pharmacol. Exp. Ther. 195, 453-464.

Loeffler, D. A., Lewitt, P. A., Demaggio, A. J., Juneau, P. L., Milbury, P. E., and Matson, W. R. (1995). Markers of dopamine depletion and compensatory response in striatum and cerebrospinal fluid. J. Neural Transm. Park Dis. Dement Sect. 9, 45-53. doi: 10.1007/BF02252962

Lovett-Barron, M., Andalman, A. S., Allen, W. E., Vesuna, S., Kauvar, I., Burns, V. M., et al. (2017). Ancestral circuits for the coordinated modulation of brain state. Cell 171, 1411.e17-1423.e17. doi: 10.1016/j.cell.2017. 10.021 
Ludwig, M., and Leng, G. (2006). Dendritic peptide release and peptidedependent behaviours. Nat. Rev. Neurosci. 7, 126-136. doi: 10.1038/ nrn 1845

Mancillas, J. R., Siggins, G. R., and Bloom, F. E. (1986). Somatostatin selectively enhances acetylcholine-induced excitations in rat hippocampus and cortex. Proc. Natl. Acad. Sci. U.S.A. 83, 7518-7521. doi: 10.1073/pnas.83.19. 7518

Martin, J., Kagerbauer, S. M., Schuster, T., Blobner, M., Kochs, E. F., and Landgraf, R. (2014). Vasopressin and oxytocin in CSF and plasma of patients with aneurysmal subarachnoid haemorrhage. Neuropeptides 48, 91-96. doi: 10.1016/j.npep.2013.12.004

Meletti, S., Lucchi, C., Monti, G., Giovannini, G., Bedin, R., Trenti, T., et al. (2017). Decreased allopregnanolone levels in cerebrospinal fluid obtained during status epilepticus. Epilepsia 58, e16-e20. doi: 10.1111/epi.13625

Moore, C. I., Carlen, M., Knoblich, U., and Cardin, J. A. (2010). Neocortical interneurons: from diversity, strength. Cell 142, 189-193. doi: 10.1016/j.cell. 2010.07.005

Nilsson, C. L., Brinkmalm, A., Minthon, L., Blennow, K., and Ekman, R. (2001). Processing of neuropeptide Y, galanin, and somatostatin in the cerebrospinal fluid of patients with Alzheimer's disease and frontotemporal dementia. Peptides 22, 2105-2112. doi: 10.1016/S0196-9781(01)00 571-X

Obien, M. E., Deligkaris, K., Bullmann, T., Bakkum, D. J., and Frey, U. (2014). Revealing neuronal function through microelectrode array recordings. Front. Neurosci. 8:423. doi: 10.3389/fnins.2014.00423

O'Connell, L. A. (2013). Evolutionary development of neural systems in vertebrates and beyond. J. Neurogenet. 27, 69-85. doi: 10.3109/01677063.2013. 789511

Olshausen, B. A., and Field, D. J. (2004). Sparse coding of sensory inputs. Curr. Opin. Neurobiol. 14, 481-487. doi: 10.1016/j.conb.2004.07.007

Orhan, F., Fatouros-Bergman, H., Goiny, M., Malmqvist, A., Piehl, F., Karolinska Schizophrenia Project (KaSP) Consortium, et al. (2017). CSF GABA is reduced in first-episode psychosis and associates to symptom severity. Mol. Psychiatry doi: 10.1038/mp.2017.25 [Epub ahead of print].

Otto, F., Illes, S., Opatz, J., Laryea, M., Theiss, S., Hartung, H. P., et al. (2009). Cerebrospinal fluid of brain trauma patients inhibits in vitro neuronal network function via NMDA receptors. Ann. Neurol. 66, 546-555. doi: 10.1002/ana. 21808

Palfreyman, M. G., Huot, S., and Wagner, J. (1982). Value of monoamine metabolite determinations in CSF as an index of their concentrations in rat brain following various pharmacological manipulations. J. Pharmacol. Methods 8, 183-196. doi: 10.1016/0160-5402(82)90073-0

Palm, G. (2013). Neural associative memories and sparse coding. Neural Netw. 37, 165-171. doi: 10.1016/j.neunet.2012.08.013

Parent, A. (1981). Comparative anatomy of the serotoninergic systems. J. Physiol. 77, 147-156.

Perez-Alcazar, M., Culley, G., Lyckenvik, T., Mobarrez, K., Bjorefeldt, A., Wasling, P., et al. (2016). Human cerebrospinal fluid promotes neuronal viability and activity of hippocampal neuronal circuits in vitro. Front. Cell Neurosci. 10:54. doi: 10.3389/fncel.2016.00054

Romigi, A., Bari, M., Placidi, F., Marciani, M. G., Malaponti, M., Torelli, F., et al. (2010). Cerebrospinal fluid levels of the endocannabinoid anandamide are reduced in patients with untreated newly diagnosed temporal lobe epilepsy. Epilepsia 51, 768-772. doi: 10.1111/j.1528-1167.2009. 02334.x

Saper, C. B., Chou, T. C., and Scammell, T. E. (2001). The sleep switch: hypothalamic control of sleep and wakefulness. Trends Neurosci. 24, 726-731. doi: 10.1016/S0166-2236(00)02002-6

Saunders, N. R., Knott, G. W., and Dziegielewska, K. M. (2000). Barriers in the immature brain. Cell Mol. Neurobiol. 20, 29-40. doi: 10.1023/A:100699180 9927

Schmidt, A. P., Bohmer, A. E., Hansel, G., Soares, F. A., Oses, J. P., Giordani, A. T., et al. (2015). Changes in purines concentration in the cerebrospinal fluid of pregnant women experiencing pain during active labor. Neurochem. Res. 40, 2262-2269. doi: 10.1007/s11064-015-1716-9

Schwarz, N., Hedrich, U. B. S., Schwarz, H., Harshad, P. A., Dammeier, N., Auffenberg, E., et al. (2017). Human cerebrospinal fluid promotes longterm neuronal viability and network function in human neocortical organotypic brain slice cultures. Sci. Rep. 7:12249. doi: 10.1038/s41598-017-12 527-9

Singer, W. (1999). Neuronal synchrony: a versatile code for the definition of relations? Neuron 24, 49-65, 111-125. doi: 10.1016/S0896-6273(00) 80821-1

Skipor, J., and Thiery, J. C. (2008). The choroid plexus-cerebrospinal fluid system: undervaluated pathway of neuroendocrine signaling into the brain. Acta Neurobiol. Exp. 68, 414-428.

Skrede, K. K., and Westgaard, R. H. (1971). The transverse hippocampal slice: a well-defined cortical structure maintained in vitro. Brain Res. 35, 589-593. doi: 10.1016/0006-8993(71)90508-7

Smith, D. E., Johanson, C. E., and Keep, R. F. (2004). Peptide and peptide analog transport systems at the blood-CSF barrier. Adv. Drug Deliv. Rev. 56, 1765-1791. doi: 10.1016/j.addr.2004.07.008

Sossi, V., De La Fuente-Fernandez, R., Holden, J. E., Doudet, D. J., Mckenzie, J., Stoessl, A. J., et al. (2002). Increase in dopamine turnover occurs early in Parkinson's disease: evidence from a new modeling approach to PET 18 F-fluorodopa data. J. Cereb. Blood Flow Metab. 22, 232-239. doi: 10.1097/ 00004647-200202000-00011

Spreux-Varoquaux, O., Bensimon, G., Lacomblez, L., Salachas, F., Pradat, P. F., Le Forestier, N., et al. (2002). Glutamate levels in cerebrospinal fluid in amyotrophic lateral sclerosis: a reappraisal using a new HPLC method with coulometric detection in a large cohort of patients. J. Neurol. Sci. 193, 73-78. doi: 10.1016/S0022-510X(01)00661-X

Strittmatter, M., Grauer, M., Isenberg, E., Hamann, G., Fischer, C., Hoffmann, K. H., et al. (1997). Cerebrospinal fluid neuropeptides and monoaminergic transmitters in patients with trigeminal neuralgia. Headache 37, 211-216. doi: 10.1046/j.1526-4610.1997.3704211.x

Swallow, J. G., Bubak, A. N., and Grace, J. L. (2016). Editorial the role of monoamines in modulating behavior. Curr. Zool. 62, 253-255. doi: 10.1093/ cz/zow046

Sykova, E. (2004). Extrasynaptic volume transmission and diffusion parameters of the extracellular space. Neuroscience 129, 861-876. doi: 10.1016/j.neuroscience. 2004.06.077

Tononi, G., and Cirelli, C. (2014). Sleep and the price of plasticity: from synaptic and cellular homeostasis to memory consolidation and integration. Neuron 81 , 12-34. doi: 10.1016/j.neuron.2013.12.025

Tremblay, R., Lee, S., and Rudy, B. (2016). GABAergic interneurons in the neocortex: from cellular properties to circuits. Neuron 91, 260-292. doi: 10. 1016/j.neuron.2016.06.033

Tsubokawa, H., and Ross, W. N. (1997). Muscarinic modulation of spike backpropagation in the apical dendrites of hippocampal CA1 pyramidal neurons. J. Neurosci. 17, 5782-5791.

van den Pol, A. N. (2012). Neuropeptide transmission in brain circuits. Neuron 76, 98-115. doi: 10.1016/j.neuron.2012.09.014

Veening, J. G., and Barendregt, H. P. (2010). The regulation of brain states by neuroactive substances distributed via the cerebrospinal fluid; a review. Cerebrospinal Fluid Res. 7:1. doi: 10.1186/1743-84 54-7-1

Vigh, B., Manzano, E., Silva, M. J., Frank, C. L., Vincze, C., Czirok, S. J., et al. (2004). The system of cerebrospinal fluid-contacting neurons. Its supposed role in the nonsynaptic signal transmission of the brain. Histol. Histopathol. 19, 607-628. doi: 10.14670/HH-19.607

Vizi, E. S., Fekete, A., Karoly, R., and Mike, A. (2010). Non-synaptic receptors and transporters involved in brain functions and targets of drug treatment. Br. J. Pharmacol. 160, 785-809. doi: 10.1111/j.1476-5381.2009. 00624.x

Wagenaar, D. A., Pine, J., and Potter, S. M. (2006). An extremely rich repertoire of bursting patterns during the development of cortical cultures. BMC Neurosci. 7:11. doi: 10.1186/1471-2202-7-11

Welch, M. J., Markham, C. H., and Jenden, D. J. (1976). Acetylcholine and choline in cerebrospinal fluid of patients with Parkinson's disease and Huntington's chorea. J. Neurol. Neurosurg. Psychiatry 39, 367-374. doi: 10.1136/jnnp.39. 4.367

Whish, S., Dziegielewska, K. M., Mollgard, K., Noor, N. M., Liddelow, S. A., Habgood, M. D., et al. (2015). The inner CSF-brain barrier: developmentally controlled access to the brain via intercellular junctions. Front. Neurosci. 9:16. doi: $10.3389 /$ fnins.2015.00016 
Wolfe, J., Houweling, A. R., and Brecht, M. (2010). Sparse and powerful cortical spikes. Curr. Opin. Neurobiol. 20, 306-312. doi: 10.1016/j.conb.2010. 03.006

Zlokovic, B. V., Jovanovic, S., Miao, W., Samara, S., Verma, S., and Farrell, C. L. (2000). Differential regulation of leptin transport by the choroid plexus and blood-brain barrier and high affinity transport systems for entry into hypothalamus and across the blood-cerebrospinal fluid barrier. Endocrinology 141, 1434-1441. doi: 10.1210/endo.141.4.7435

Zubenko, G. S., Marquis, J. K., Volicer, L., Direnfeld, L. K., Langlais, P. J., and Nixon, R. A. (1986). Cerebrospinal fluid levels of angiotensin-converting enzyme, acetylcholinesterase, and dopamine metabolites in dementia associated with Alzheimer's disease and Parkinson's disease: a correlative study. Biol. Psychiatry 21, 1365-1381. doi: 10.1016/0006-3223(86)90328-8
Conflict of Interest Statement: The authors declare that the research was conducted in the absence of any commercial or financial relationships that could be construed as a potential conflict of interest.

The reviewer GK and handling Editor declared their shared affiliation.

Copyright (c) 2018 Bjorefeldt, Illes, Zetterberg and Hanse. This is an open-access article distributed under the terms of the Creative Commons Attribution License (CC BY). The use, distribution or reproduction in other forums is permitted, provided the original author(s) and the copyright owner are credited and that the original publication in this journal is cited, in accordance with accepted academic practice. No use, distribution or reproduction is permitted which does not comply with these terms. 